\title{
Classification of Human Ear by Extracting Hog Features and Support Vector Machine
}

\author{
Naveena M. \\ Scientist, HPC, UPE \\ University of Mysore \\ Mysore- 570006
}

\author{
G. Hemantha Kumar \\ Vice Chancellor \\ University of Mysore \\ Mysore-570006
}

\begin{abstract}
The approaches proposed by eminent researchers have been discussed in the previous sections. Survey on the researches says that, it defines the uniqueness of a given person. And it is possible in the biometric authentication to have collisions between two people who have completely biometric character. Gabor filter can represents the frequency and orientation of similar to those of the human visual System, and they have been found to be particularly appropriate for texture representation and as well as discrimination. There will be a great research on the HOG, it is purely gradient based and captured the object shape information; it can be used to extract the global feature. HOG compute edge gradient of whole image and find orientation of each pixel so it can generate histogram easily. Many of them used the Support vector machine for classification because it avoids the over fitting, and it can built kernel and also it is an approximation to a bound on the test error rate. The theory behind SVM suggests that it should a good idea.
\end{abstract}

\section{Keywords}

Support Vector Machine, HOG

\section{INTRODUCTION}

Biometrics refers to the recognition of individuals of person based on physiological and behavioral characteristics. Ear is a new workable class in biometric. The ear is one such biometric because it is found to be unique even among twins. Public safety and national security enhance the needs for biometric techniques, which are among the most secure and accurate authentication tools. Ear biometric applications are interesting especially in crime investigation. In this paper we present the basics of using ear as biometric for person identification.

This paper presents the novel techniques for automated human ear recognition system. There are four steps of the proposed algorithm. In the first step preprocessing is applied on the ear image which includes resizing, RGB to Gray conversion and Identify the texture variations using Gabor filter. In the second step feature of the ear are extracted using Histogram of Gradients. In the third step of proposed system feature matching is done using the SVM classifiers which give good results for design matching.

Biometric: The word "biometrics" is derived from the Greek words 'bios' and 'metric' which means life and measurement respectively. Biometric verification means a person can be uniquely identified by evaluating one or more distinguishing biological traits. There are two main types of biometric identifiers. Physiological characteristics: The shape or composition of the body. Examples are fingerprints, DNA, face, hand, retina or ear features and odor. And Behavioral characteristics: The behavior of a person. Behavioral characteristics are related to the pattern of the behavior of a person, such as typing rhythm, gait, gestures and voice.

Ear Biometrics: Human ear contains large amount of specific and unique features that allows for human identification. Biometric systems have become very essential components in almost all security aspects. Ear has gained much attention in recent years as it has been found to be a good and reliable biometrics for human verification and identification. Reason behind the ear biometrics gaining popularity is that ears are remarkably consistent. Because ear biometric has a lot of benefits over other biometric technologies such as face, retinal scans, iris and fingerprints. Unlike the faces, they do not change shape with different expressions or age, and remain fixed in the middle of the side of the head against a predictable background. And the image is obtained easily, and also it can be taken from a distance without the mutual effort from the distinctive.

Anatomy of the Ear: The anatomy of the external ear, also known as the auricle or pinna, is complex and remarkably inaccurately described by most authors. The major landmarks of the external ear are depicted in Figure 1. The external ear consists of skin, cartilage, and six intrinsic muscles. The anatomy of the various components of the ear is described below, and illustrations are shown each time in the section describing the various features of the components.

Need for Ear Biometrics: The human ear is a stable structure that does not change much in shape with the age and with facial expressions. Uniqueness of outer ear shape that do not change because of emotion etc. Ears are remarkably consistent, unlike faces, they do not change shape with different expressions or age. And crime investigation is interested in using ear identification.

\section{LITERATURE SURVEY}

\section{Previous work on Ear Biometric:}

The physiological shape of the outer ear was initially founded by the French criminologist Bertillon by American police Officer Alfred Iannarelli. He has created Iannarelli system based on the twelve measurement of the ear. Alfred Iannarelli practice only with the right ear of the people. To determine the picture, the range between each of twelve numbered areas are measured and assigned an integer value. But the concept of Iannarelli system is not acceptable for device perception. And also he studied on twins. In this case the hypothesis of ear is unique. In the identical twins the ear physiological feature are similar but it not identical.

\section{Previous work on Gabor feature:}

Yaqubi et al. [2008] this paper is deals with the feature extraction method based on a set of Gabor filters followed by a maximization operation over multiple scales and positions. This method is motivated by a quantitative model of the visual cortex. Then they used Support Vector Machine (SVM) for 
ear classification. They obtained a recognition rate of $75 \%$ on a subset of the USTB ear database where 180 ear images were manually extracted from 60 subjects. Fields et al. [1960] made an experiment to recognize newborn babies in hospitals. They optically estimate the 206 sets of ear photographs, and concluded that the morphological constancy of the ear can be used to establish the identity of the newborn.

\section{Previous work on HOG:}

Histograms of Oriented Gradients for Human Detection by Navneet Dalal and Bill Triggs. This research shows that usage of locally normalized histogram of gradient orientations features similar to SIFT descriptors, also in a heavy overlapping grid gives very good results for person detection, and also it reduces the false positive rates by more than an order of magnitude relative to the best Haar wavelet based detector from. They work on the influence of various descriptor parameters and concluded that fine-scale gradient, fine orientation binning, relatively coarse spatial binning, and high-quality local contrast normalization in overlapping descriptor blocks are all important for good performance. And they introduced a new and more challenging pedestrian database, which is publicly available.

\section{Previous work on classification:}

To have a successful recognition of ear by a set of features a trained Machine learning algorithm is essential to categories the given ear image into its respective categories. Several methods concerned to machine learning algorithm have been proposed. Schuldt, Laptev and Caputo [16], G. Zhu, C. Xu, et al proposed a paper on recognizing human actions using Support Vector Machine (SVM) for the extracted features, in which local space-time features capture local events in video and can be adapted to the size, the frequency and the velocity of moving patterns. Geeta Maurya presented a method to detect human actions using neural network by pattern recognition. They introduced an application to pattern recognition using spiking neurons which holds biological evidences and enhance the performance of existing by using back propagation algorithm.

\section{OBJECTIVE}

The main aim is to develop a robust biometric system using the human ear.

\section{ADVANTAGES}

\section{Advantages of biometrics:}

a) Increase security - Provide a convenient and lowcost additional tier of security.

b) Reduce fraud by employing hard-to-forge technologies and materials. For e.g. minimize the opportunity for ID fraud, buddy punching.

c) Eliminate problems caused by lost IDs or forgotten passwords by using physiological attributes. For e.g. prevent unauthorized use of lost, stolen or "borrowed" ID cards.

d) Reduce password administration costs.

e) Replace hard-to-remember passwords which may be shared or observed.

f) Integrate a wide range of biometric solutions and technologies, customer applications and databases into a robust and scalable control solution for facility and network access.

\section{Advantages of Ear Biometrics:}

a) Ear images can be easily taken from a distance and without knowledge of the examined person.

b) Ear does not change during human life and face changes more significantly with age, cosmetics, facial hair, hair styling, emotions expression. Color distribution is more uniform.

c) Ear is also smaller than face then it is possible to work faster and more efficiently with the images with the lower resolution.

d) Ear has all the properties that a biometric traits should have that is, Uniqueness, Universality, Performance and collectability.

3. Advantages of SVM:

a) The computational complexity of SVMs does not depend on the dimensionality of the input space.

b) SVM training always finds a global minimum and their simple geometric interpretation provides fertile ground for further investigation.

c) The SVM approach does not attempt to control model complexity by keeping the number of features small

d) In comparison with traditional multilayer perception neural networks that suffer from the existence of multiple local minima solutions, convexity is an important and interesting property of nonlinear SVM classifiers.

\section{PROPOSED METHODOLOGY}

Standard dataset: The database can be collected from the AMI Ear Database. The image has been taken from indoor and all of them in the age range between 19 to 65 years. All images taken using a NIKEN D 100 camera under same lighting condition with distance about $2 \mathrm{~m}$ from camera.

Pre-Processing: In general, the input signal is affected by unwanted signal such as, noise in the capturing device, lighting variation, changes in background clutters like tress and so on, due to which the required details cannot to be extracted satisfactorily. Hence, the input image needs to be pre-processed to remove unwanted signals in order to preserve the required details. This process is done before feature extraction.

Feature Extraction: The variation in the texture can be detected using the Gabor filter, is fed into feature extraction technique using the Histogram of Oriented Gradients (HOG). HOG is a feature descriptor used to detect objects in computer vision and image processing. This technique counts occurrences of gradient orientation in localized portions of an image - region of interest. This feature is necessary to train and test the algorithm which is used to recognize the ear.

Supervised Learning: It is one of the types of machine learning. The supervised learning algorithms are trained on Labeled example i.e., input where desired output is known. The supervised learning algorithm attempts to generalize a function or mapping from inputs to outputs which can then be used speculatively to generate an output for previously unseen inputs.

\section{DATASET COLLECTION}

The database of 700 images has been sequentially numbered for every subject with an integer identification number. The 
resolution of these images is $492 \times 702$ pixels and all these images are available in jpeg format. Few examples of images from the AMI Ear Database are reproduced in the following image set.

a) RGB images
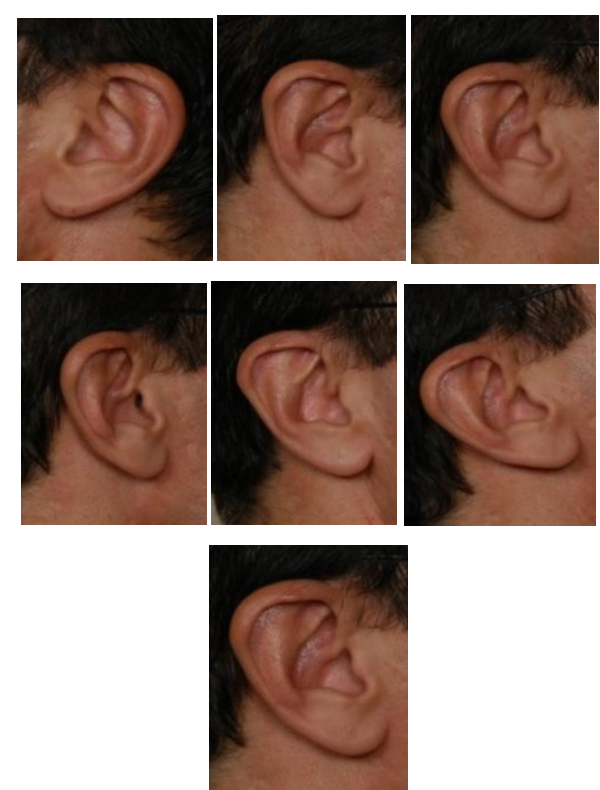

Fig 1: Standard Data Sets

b) Pre-Processing: RGB to Gray
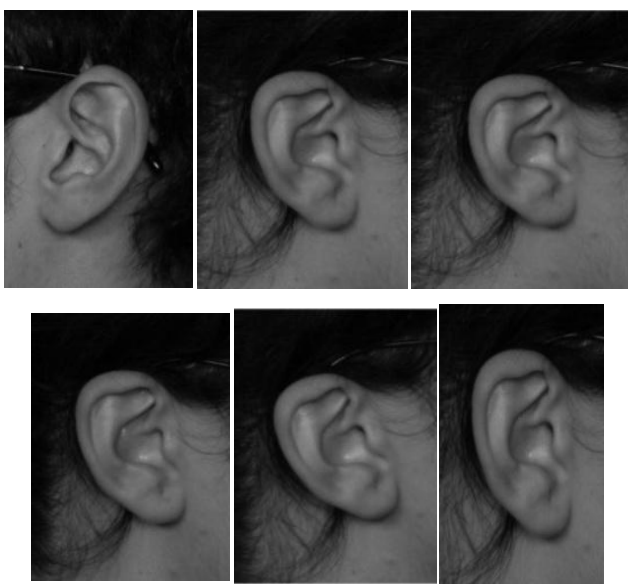

Fig2: RGB to Gray Outputs

c) Gabor filters
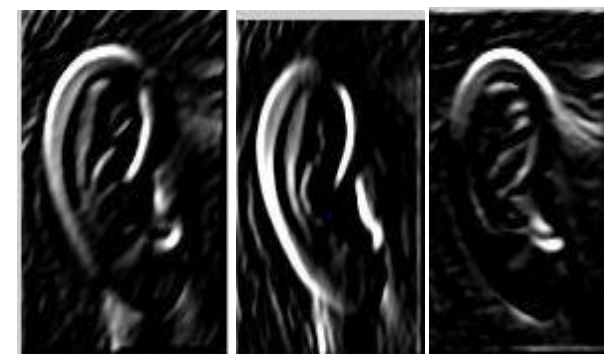
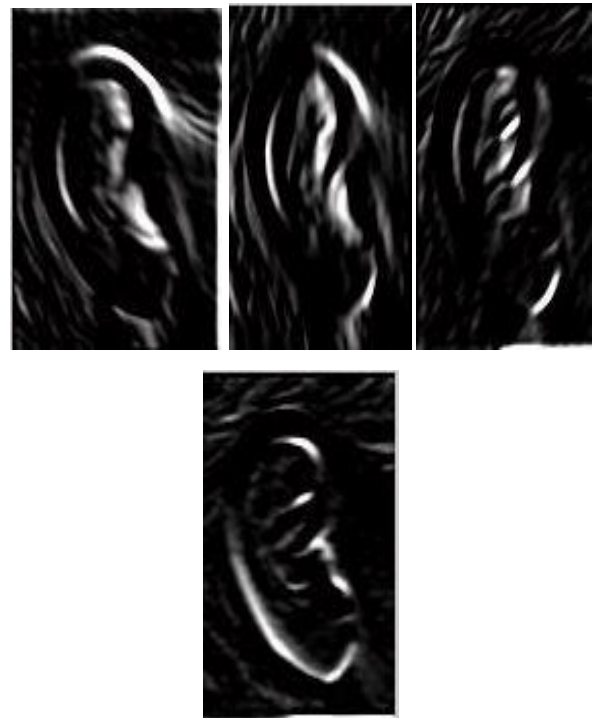

Fig3: Gabor outputs

\section{IMPLIMENTATION RESULTS}

The depicted figures represent the outputs at different stages of the implementation process. (a) RGB input image, (b) Gray image, (c) extracted Gabor feature from gray image, (d) detected class from the extracted features.
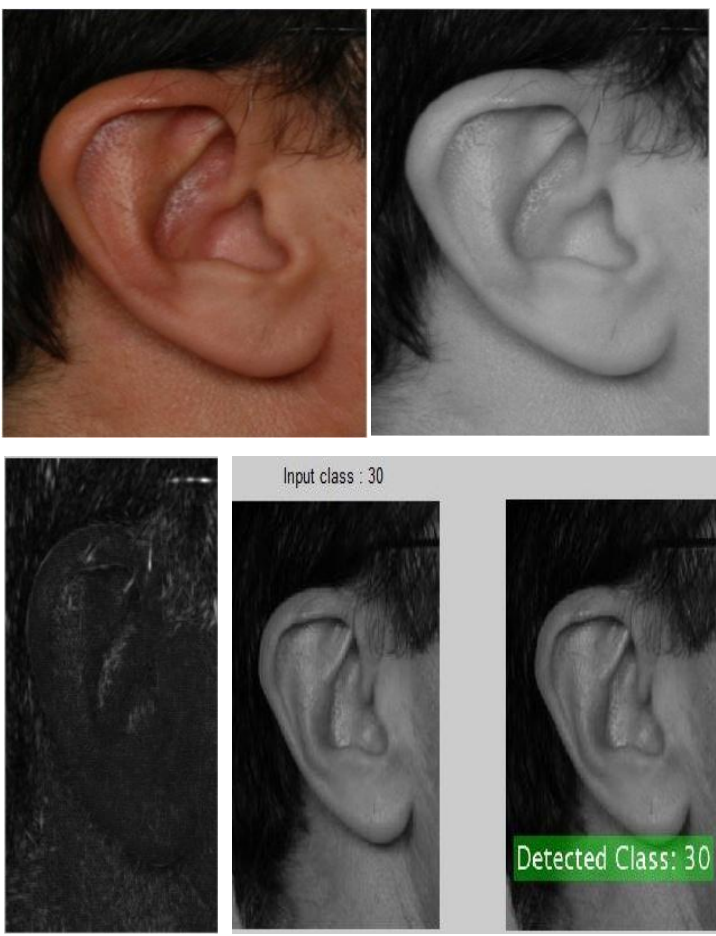

Fig 4: Implementation results 


\section{Confusion matrix for SVM classifier}

\begin{tabular}{|c|c|c|c|c|c|c|c|c|c|c|}
\hline $\begin{array}{c}\text { SVM } \\
\text { classifier }\end{array}$ & P1 & P2 & P3 & P4 & P5 & P6 & P7 & P8 & P9 & P10 \\
\hline P1 & $\mathbf{1}$ & 0 & 0 & 0 & 0 & 0 & 0 & 0 & 0 & 0 \\
\hline P2 & 0 & $\mathbf{1}$ & 0 & 0 & 0 & 0 & 0 & 0 & 0 & 0 \\
\hline P3 & 0 & 0 & $\mathbf{1}$ & 0 & 0 & 0 & 0 & 0 & 0 & 0 \\
\hline P4 & 0 & 0 & 0 & $\mathbf{1}$ & 0 & 0 & 0 & 0 & 0 & 0 \\
\hline P5 & 0 & 0 & 0 & 0 & $\mathbf{1}$ & 0 & 0 & 0 & 0 & 0 \\
\hline P6 & 0 & 0 & 0 & 0 & 0 & $\mathbf{1}$ & 0 & 0 & 0 & 0 \\
\hline P7 & 0 & 0 & 0 & 0 & 0 & 0 & $\mathbf{1}$ & 0 & 0 & 0 \\
\hline P8 & 0 & 0 & 0 & 0 & 0 & 0 & 0 & $\mathbf{1}$ & 0 & 0 \\
\hline P9 & 0 & 0 & 0 & 0 & 0 & 0 & 0 & 0 & $\mathbf{1}$ & 0 \\
\hline P10 & 0 & 0 & 0 & 0 & 0 & 0 & 0 & 0 & 0 & $\mathbf{1}$ \\
\hline
\end{tabular}

\section{Average class accuracy on AMI database}

\begin{tabular}{|c|c|}
\hline Classifier name & Accuracy \\
\hline SVM & 100 \\
\hline
\end{tabular}

From the confusion matrix for the tested ears average class accuracy obtained for SVM classifiers is $100 \%$ on the AMI dataset.

\section{CONCLUSION}

In this report a Ear biometric system for verification of person are presented. We have tested our method on Standard dataset. This method based approach can be extended to occlusion ear image. We used supervised learning algorithm (SVM) to train and test the classifier to obtain better results. Here we obtain the up to $100 \%$ of result using the SVM for training and testing purpose.

\section{FUTURE WORK}

Ear biometric has a scope in the research field of computer visions under which there is a scope to develop many real time algorithms. In the work the proposed algorithm has certain limitations like, it is the image is taken under limited environmental conditions i.e., homogeneous lightning condition, same distance between the camera and a person.

The proposed algorithm is tested with offline images. Our future endeavor will be to make our system more accurate and construct a general approach to deal with video surveillance and it can test with online images. If the images are taken from different lightning condition and variations in the distance while capturing the image, the accuracy will reduce. If this drawback is overcome, then the algorithm is more suitable for real time images input.

\section{REFERENCES}

[1] Ear Biometrics: A Survey of Detection, Feature Extraction and Recognition Methods A. Pflug, Christoph Busch July 2, 2012

[2] Ear Biometrics in Human Identification System V.K. N.Kumar 1 and B, Srinivasan 21 Assistant Professor,PG \& Research Department of Computer Science, Gobi Arts $\&$ Science College,Erode District, Tamil Nadu, India.

[3] Person Identification Using Ear Biometrics M.Raman, Md.Islam Nazmul Islam Bhuiyan, Bulbul Ahmed, Md.
Aminul Islam Computer Science and Engineering Discipline, Khulna University,Khulna-9208, Bangladesh.

[4] Fusion of Ear and Soft-biometrics for Recognition of Newborn Tiwari, A Singh, S Kumar Singh Department of Computer Engineering Institute of Technology, Hindu University Varanasi, India-221005

[5] Histograms of Oriented Gradients for Human Detection Navneet Dalal and Bill Triggs INRIA Rhône-Alps, 655 avenue de l'Europe, Montbonnot 38334,France.

[6] BoF meets HOG: Feature Extraction based on Histograms of Oriented p.d.f Gradients for Image Classification Takumi Kobayashi National Institute of Advanced Industrial Science and Technology 1-1-1 Umezono, Tsukuba, Japan.

[7] A Multimodal SVM Approach for Fused Biometric Recognition Geethu S Kumar Jyothirmati Devi Department of Computer Science and Engineering College of Engineering, Chengannur, Kerala

[8] Support Vector Machines Max Welling Department of Computer Science University of Toronto 10 King's College Road Toronto, M5S 3G5 Canada welling@cs.toronto.edu

[9] Comparison of Texture Features Based on Gabor Filters Simona E. Grigorescu, Nicolai Petkov, and Peter Kruizinga.

[10] Gabor wavelet transform and its application Wei-lun Chao R98942073

[11] Texture Segmentation Using Gabor Filters Vincent Levesque vleves@cim.mcgill.ca Center for Intelligent Machines, McGill University December 6, 2000.

[12] A Survey on Human Ear Recognition Suvarnsing Bhable1S.Tharewal Dr. K.V.Kale Department of Computer Science and Information Technology Dr. Babasaheb Ambedkar Marathwada University, Aurangabad. 